\title{
Autoconcepto académico, motivación escolar e implicación escolar del alumnado universitario de República Dominicana y España ${ }^{1}$
}

\author{
Iván De Jesús Moronta Tremols, Arantzazu Rodríguez-Fernández y Oihane Fernández-Lasarte \\ Universidad del País Vasco - España
}

\begin{abstract}
RESUMEN
Esta investigación examina las diferencias en el autoconcepto académico, la motivación escolar y la implicación escolar en función del sexo y el país en universitarios de República Dominicana y España, así como la dirección de la relación de estas variables en estos países. Se utilizó una muestra de 1101 estudiantes de Universidades públicas y privadas de Santo Domingo y País Vasco, con edades de 18 a 56 años, de los que 567 eran de República y 534 de España. Los instrumentos utilizados han sido el (AUDIM) para el autoconcepto, el (CEAM II) para la motivación y el (SEM) para la implicación escolar. Los resultados adquiridos señalan que: (a) que las mujeres muestran mejor autoconcepto académico verbal y los hombres en el matemático en ambos países. (b) en motivación general existe diferencia a favor de las mujeres en España. (c) existe diferencia a favor de las mujeres en la implicación en ambos países. (d) existe diferencia a favor de República en todas las dimensiones de estas variables. (e) la direccionalidad es explicativa en ambas direcciones en ambos países.
\end{abstract}

Palabras Clave: Autoconcepto académico, motivación escolar, implicación escolar.

\section{Academic self-concept, school motivation and school engagement in university students from the Dominican Republic and Spain}

\section{ABSTRACT}

This research studies differences in academic self-concept, school motivation and school engagement between university students from the Dominican Republic and Spain, considering gender and country, as well as the direction of these variables in both countries. The sample considers 1101 students from 18 to 56 years old, from public and private universities among Santo Domingo and the Basque Country, of which 567 were from the Dominican Republic and 534 from Spain. The instruments implemented were the (AUDIM) for the self-concept, the (CEAM II) for the school motivation and the (SEM) for the school engagement. The results obtained indicate that: (a) in both countries women show better verbal academic self-concept and men in math. (b) In Spain there is a difference in the general motivation on behalf of women. (c) In both countries there is a difference for the implication on behalf of women. (d) there is a difference in all the dimensions of these variables on behalf of the Dominican Republic. (e) In both countries Directionality is explicative for both directions.

Keywords: Academic self-concept, school motivation, school engagement.

\section{Introducción}

Blumenfeld, Fredicks y Paris (2004) fueron los primeros en establecer un modelo tridimensional para la implicación escolar, compuesto por: una faceta conductual, entendida como la participación en el centro educativo; una faceta psicológica o sentimiento de identificación que muestra el alumnado con el centro; y una faceta cognitiva, referida a los cambios psicológicos del alumnado en su manera de aprender, así como a su capacidad de mejorar las habilidades meta-cognitivas; modelo teórico que autores posteriores han corroborado (González y Verónica-Paoloni, 2015).

Teniendo en cuenta esta naturaleza interna tridimensional de la implicación escolar, se podría definir como el compromiso o participación del estudiante en su entorno escolar (Ros, 2009) o como el grado en que el alumnado se compromete con la escuela para aprender (Simon-Morth y Chen, 2009).

Y dada la importancia del papel que la implicación escolar juega en los centros educativos (Christenson, Reschly y Wylie, 2012), se está analizando con mayor importancia no sólo cómo

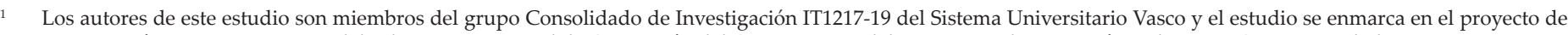
investigación EDU2017-83949-P del subprograma estatal de Generación del Conocimiento del Ministerio de Economía, Industria y Competitividad.
} 
los estudiantes se implican en los procesos educativos dentro de sus centros escolares, sino también qué variables se relacionan o influyen en que el alumnado se implique en mayor grado en la vida escolar (Ramos-Díaz, Rodríguez-Fernández, Fernández-Zabala, Revuelta y Zuazagoirtia, 2016; Ros, 2009).

A mediados del siglo XX autores señalaban la necesidad de tener en cuenta las características del alumnado en el estudio del ajuste escolar (implicación escolar), entre ellas el autoconcepto (Dimont, 1990) y la motivación (Deci y Ryan, 2000). Estudios más recientes lo corroboran al haber hallado que entre las principales variables que influyen en la implicación escolar se encuentran el autoconcepto (Inglés, Martínez-Monteagudo, García-Fernández, Valle y Castejón, 2014; Rodríguez-Fernández, Droguett y Revuelta, 2012) y la motivación (Dettmers, Lüdtke, Trautwein, Kunter y Baumert, 2010).

En otros estudios van más allá y establecen una direccionalidad en dicha relación (Christenson et al., 2012), evidenciando que aspectos ambientales y psicológicos, como el autoconcepto y/o la motivación (Clarks, Clemes y Bean, 2000; Dettmers et al., 2010), puede llegar a influir en el nivel de implicación en la escuela (Clarks et al., 2000).

Cabe señalar que en las últimas décadas se han estado realizando un sinnúmero de investigaciones sobre el autoconcepto. A pesar de que no existe una definición univocoa sobre este constructo, la más utilizada podría ser que el concepto que se tiene de sí mismo es la combinación de imágenes, conocimientos, percepciones y representaciones que un sujeto posee de su persona (Shavelson, Hubner, y Stanton, 1976; García y Musitu, 2009).

Al intentar comprender cómo el alumnado se adecúa al entorno escolar, Birch y Ladd (1996) señalan que el autoconcepto es vital en el desarrollo adaptativo de un individuo con su entorno, algo corroborado por autores como Fuentes, García, Gracia y Lila (2011) y Rodríguez-Fernández et al. (2012) quienes señalan que el autoconcepto forma parte de ese conjunto de variables psicológicas que desempeña un rol importante en la implicación escolar.

Autores como Garaigordobil y Durá (2006) afirman que los adolescentes con mayor nivel de autoconcepto muestran una cantidad superior de conductas efectivas, y una mayor implicación escolar (Martínez-Antón, Buelga y Cava, 2007). De igual forma, se ha comparado a personas con un alto nivel de autoconcepto con otros que poseen un bajo nivel de autoconcepto, pudiendo observarse que los de mayor nivel son apreciados como cooperativos y populares en su centro escolar, es decir, personas más implicadas en temas escolares (Hay, Ashman y Van-Kraayenoord, 1998).

En cuanto a la motivación, los distintos investigadores concuerdan en que resulta fundamental para la implicación escolar a la hora de realizar las tareas escolares; algunos señalan que el tipo de motivación que presente el alumnado estará relacionada con la calidad de implicación demostrada (Dettmers et al., 2010). Pero, en cambio, no existe acuerdo sobre la dirección de dicha relación. Algunos autores defienden que es la motivación la que incide sobre la implicación escolar (Corno, 2000; Xu y Corno, 1998), mientras que otros defienden el sentido contrario: la calidad de la implicación es la que determina el tipo de motivación (Dettmers et al., 2010) o que incluso es la implicación en las tareas escolares la que indica el nivel de motivación escolar (Bempechat, 2004).

A su vez, los estudios previos sobre las diferencias en el autoconcepto señalan que, en cuanto al sexo los hombres poseen una mejor puntuación en autoconcepto académico que las mujeres, si bien en el autoconcepto verbal las chicas conseguían mejores puntuaciones que los chicos_(Inglés, Pastor, Torregrosa, Redon- do y García, 2009; Fernández-Zabala, Goñi, Rodríguez-Fernández y Goñi, 2015). Otros a su vez señalan que no existe diferencia alguna (Amezcua y Pichardo, 2000). No obstante, en un estudio realizado en la República Dominicana se obtuvieron datos contrarios a estos: las chicas muestran un mejor concepto de sí que los chicos (Barca, Fernández y Mejía, 2011).

En un estudio realizado por Cerezo y Casanova (2004) en relación a las diferencias en motivación en función del sexo, los resultados señalan que las mujeres poseen un menor nivel de motivación extrínseca (la que se original por factores externos). Sin embargo, otra investigación señala que los hombres tenían mejor motivación extrínseca, mientras que las mujeres poseían una mejor motivación intrínseca (la que se da originalmente en el interior) (Vallerand y Bissonnette, 1992). Otro estudio enfocado en la misma línea señala de modo general, que las chicas se encuentran más motivadas que los chicos sin importar su edad (Núñez, González-Pienda, García, González-Pumariega y García, 1998), pero nada se sabe del comportamiento de esta variable en República Dominicana.

Ahora bien, es notable la carencia de estudios que analicen estas variables en estudiantes universitarios en República Dominicana, también destaca la ausencia de trabajos comparativos entre el alumnado de ese país con universitarios españoles.

Aunque, como se ha visto, existen estudios que analizan la asociación de la implicación escolar con el autoconcepto académico y la motivación de forma separada, no se conoce si esas relaciones se mantienen para el alumnado universitario de República Dominicana; y tampoco se conoce si en función del sexo los niveles de esas variables difieren para ambos países. De igual forma, no se conoce la direccionalidad de dichas relaciones ¿es el autoconcepto y la motivación académica los que inciden en la implicación o, por el contrario, ¿es el grado de implicación escolar quien determina las autopercepciones del estudiante y su grado de motivación escolar? Por tanto, el objetivo de esta investigación es doble:

1- Analizar las diferencias en autoconcepto académico, motivación escolar e implicación escolar en función del país (República Dominicana y España) y del sexo.

2- Aclarar la direccionalidad de la relación entre autoconcepto académico, motivación escolar e implicación escolar en estudiantes universitarios de ambos países.

\section{Método}

\section{Participantes}

Se eligió a los participantes siguiendo el método de muestreo incidental, obteniendo una muestra total de 1101 sujetos de edades comprendidas entre los 18 y los 56 años de edad ( $M=24,28$; $\mathrm{DT}=5,55)$. Todos los participantes eran estudiantes de diferentes Universidades privadas y públicas de República Dominicana (Santo Domingo) y España (País Vasco) con una muestra de 567 alumnos de República (51,50\%) y 534 estudiantes $(48,50 \%)$ de España.

\section{Instrumentos de medidas}

Para analizar las variables sociopersonales, se realizaron preguntas como el sexo y el país para conocer la cantidad de hombres y mujeres participantes y el país de residencia.

El autoconcepto académico se valora mediante el cuestionario de Autoconcepto Dimensional (AUDIM-33) de Fernández-Zabala et al., (2015). Este cuestionario evalúa tanto el autoconcepto ge- 
neral, como el autoconcepto académico verbal, el académico matemático, la habilidad y condición física, atractivo físico, fuerza física, honradez, ajuste emocional, autonomía, autorrealización, responsabilidad social y competencia social, pero para este estudio únicamente se utilizan las dimensiones correspondientes al autoconcepto académico verbal y al académico matemático con un total de 8 ítems (4 por cada dimensión). Está compuesto por 33 ítems a los que se responde en una escala Likert. El cuestionario presenta un índice de fiabilidad de $\alpha=, 81$ para la muestra de República Dominicana y lo mismo es para la muestra en España $\alpha=, 81$.

Para evaluar la motivación escolar se utiliza el Cuestionario de Estrategias de Aprendizaje y Motivación (CEAM II de Pintrich, Roeser y De Groot, 1994). El cuestionario consta de 31 ítems que miden seis dimensiones motivacionales: autoeficacia, ansiedad, metas extrínsecas, metas intrínsecas, creencia de control y valor de la tarea, de las cuales para este estudio se utilizan las metas extrínsecas y las metas intrínsecas, y una sumatoria de la extrínseca e intrínseca como motivación general. Él CEAM presenta una fiabilidad en conjunto de la escala de entre $\alpha=, 84$ y $\alpha=, 86$ (González, Roces, y Tourón, 1995; Ruiz de Azúa, 2012). Para esta investigación el Alfa de Cronbach fue de $\alpha=, 91$ en República Dominicana, mientras que en España la consistencia interna fue de $\alpha=, 88$.

Para medir la implicación escolar se empleó la Spanish Version of the School Engagement Measurement (SEM; Ramos-Díaz, Rodríguez-Fernández, y Revuelta, 2016), compuesto por 19 ítems de los cuales 5 ítems se refieren a la implicación comportamental; 6 ítems se refieren a la implicación emocional; y 8 ítems miden la implicación cognitiva, y para este estudio la fiabilidad total fue de $\alpha=, 84$ para la República Dominicana y de $\alpha=, 75$ para la muestra tomada en España.

\section{Procedimiento}

Tras la puesta en contacto con las diferentes Universidades y la consecución de la autorización pertinente por parte de dichas instituciones, se procedió a la entrega de un consentimiento informado a cada participante en la investigación. Se continuó con la administración de los cuestionarios dentro del horario de clase y en las propias aulas, de forma que se aplicaba al mismo tiempo a todo el alumnado. El tiempo de respuesta al conjunto de cuestionarios osciló entre los 40 y los 50 minutos. Se aseguró el anonimato de las respuestas, así como la participación totalmente voluntaria a fin de reducir sesgos en las respuestas de los participantes que obstaculizaran la validez de los resultados obtenidos.

\section{Análisis estadísticos}

Los análisis de datos se realizaron mediante el programa estadístico SPSS 25. Se comenzó realizando una corrección de valores perdidos por imputación múltiple. Luego se procedió a hacer un análisis de diferencias de medias (t de Student) para un contraste de dos medias independientes. Finalmente, se estudió la capacidad de predicción del autoconcepto académico y la motivación escolar sobre cada una de las dimensiones de la implicación escolar, y viceversa por medio de regresiones lineales utilizando el método de pasos hacia adelante y hacia atrás, y con un nivel de confianza de $95 \%$.

\section{Resultados}

El primer objetivo trata de analizar las diferencias en el autoconcepto académico, motivación escolar e implicación escolar tanto en función del país, como del sexo en España y República Dominicana. Para ello se utilizó el análisis de medias simples prueba $(\mathrm{T})$ tal y como se puede comprobar en las tablas 1 y 2.

Tabla 1.

Diferencias en el Autoconcepto Académico, Motivación Escolar e Implicación Escolar según el Sexo en ambos países.

\begin{tabular}{|c|c|c|c|c|c|c|c|c|c|}
\hline \multicolumn{6}{|c|}{ República Dominicana } & \multicolumn{4}{|c|}{ España } \\
\hline & Sexo & $\mathbf{N}$ & M (DT) & $T$ & p & $\mathbf{N}$ & M (DT) & $T$ & $\mathrm{p}$ \\
\hline A.A.V. & $\begin{array}{l}\text { M. } \\
\text { H. }\end{array}$ & $\begin{array}{l}436 \\
129\end{array}$ & $\begin{array}{l}17,15(3,36) \\
16,12(3,31)\end{array}$ & 3,09 & ,002 & $\begin{array}{l}289 \\
237\end{array}$ & $\begin{array}{l}15,37(3,14) \\
14,24(3,51)\end{array}$ & 3,85 & ,000 \\
\hline A. A. M. & $\begin{array}{l}\text { M. } \\
\text { H. }\end{array}$ & $\begin{array}{l}436 \\
129\end{array}$ & $\begin{array}{l}13,76(3,94) \\
14,88(3,29)\end{array}$ & $-3,22$ & ,001 & $\begin{array}{l}289 \\
237\end{array}$ & $\begin{array}{l}12,39(4,88) \\
13,59(4,10)\end{array}$ & $-3,04$ & ,002 \\
\hline A. A.G. & $\begin{array}{l}\text { M. } \\
\text { H. }\end{array}$ & $\begin{array}{l}436 \\
129\end{array}$ & $\begin{array}{l}30,92(5,66) \\
30,99(5,13)\end{array}$ &,- 14 & ,887 & $\begin{array}{l}289 \\
237\end{array}$ & $\begin{array}{l}27,76(5,35) \\
27,83(5,09)\end{array}$ &,- 13 & ,891 \\
\hline M. E. & $\begin{array}{l}\text { M. } \\
\text { H. }\end{array}$ & $\begin{array}{l}436 \\
129\end{array}$ & $\begin{array}{l}22,04(5,09) \\
21,95(5,39)\end{array}$ & ,18 & ,857 & $\begin{array}{l}289 \\
237\end{array}$ & $\begin{array}{l}16,87(5,41) \\
16,10(5,06)\end{array}$ & 1,67 & ,094 \\
\hline M. I. & $\begin{array}{l}\text { M. } \\
\text { H. }\end{array}$ & $\begin{array}{l}436 \\
129\end{array}$ & $\begin{array}{l}16,34(3,57) \\
16,60(3,40)\end{array}$ &,- 75 & 451 & $\begin{array}{l}289 \\
237\end{array}$ & $\begin{array}{l}15,32(2,88) \\
15,31(2,53)\end{array}$ &, 04 & ,968 \\
\hline M. G. & $\begin{array}{l}\text { M. } \\
\text { H. }\end{array}$ & $\begin{array}{l}436 \\
129\end{array}$ & $\begin{array}{l}165,28(26,67) \\
163,41(27,44)\end{array}$ & 69 & ,489 & $\begin{array}{l}289 \\
237\end{array}$ & $\begin{array}{l}147,58(20,64) \\
143,00(20,23)\end{array}$ & 2,55 & ,011 \\
\hline I. Co. & $\begin{array}{l}\text { M. } \\
\text { H. }\end{array}$ & $\begin{array}{l}436 \\
129\end{array}$ & $\begin{array}{l}19,85(1,85) \\
19,29(2,05)\end{array}$ & 2,75 & ,006 & $\begin{array}{l}289 \\
237\end{array}$ & $\begin{array}{l}19,19(1,55) \\
18,55(1,70)\end{array}$ & 4,47 & ,000 \\
\hline I. E. & $\begin{array}{l}\text { M. } \\
\text { H. }\end{array}$ & $\begin{array}{l}436 \\
129\end{array}$ & $\begin{array}{l}24,39(4,23) \\
24,23(4,36)\end{array}$ & ,35 & ,721 & $\begin{array}{l}289 \\
237\end{array}$ & $\begin{array}{l}22,15(3,51) \\
20,90(3,34)\end{array}$ & 4,16 & ,000 \\
\hline I. C. & $\begin{array}{l}\text { M. } \\
\text { H. }\end{array}$ & $\begin{array}{l}436 \\
129\end{array}$ & $\begin{array}{l}28,95(6,29) \\
28,93(6,06)\end{array}$ & ,02 & ,978 & $\begin{array}{l}289 \\
237\end{array}$ & $\begin{array}{l}24,27(4,46) \\
24,08(4,84)\end{array}$ & ,44 & 657 \\
\hline
\end{tabular}

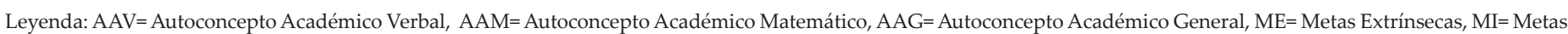
Intrínsecas, $\mathrm{MG}=$ Motivación General, ICo= Implicación Comportamental, IE= Implicación Emocional, IC= Implicación Cognitiva.

En función del sexo en cada país, la comparación de medias simple permite afirmar la existencia de diferencias significativas tanto en República Dominicana como en España en el autocon- cepto académico verbal (República: $t=3,09, p<, 01$; España: $t=3,85$, $p<, 001)$ favorable a las mujeres, en el autoconcepto académico matemático a favor de los hombres (República: $t=-3,22, p<, 01$; Es- 
paña: $t=-3,04, p<, 01)$, en la implicación comportamental a favor de las mujeres (República: $t=2,75, p<, 01$; España: $t=4,47, p<$ $, 001)$. Ahora bien, en las variables motivación general $(t=2,55, p<$ ,05) e implicación emocional $(t=4,16, p<, 001)$ únicamente existen diferencias estadísticamente significativas en España en ambos casos favorables a los universitarios varones. En el resto de va- riables (autoconcepto académido general, ambos tipos de motivación e implicación cognitiva), no se han encontrado medias estadísticamente diferentes entre ambos sexos en ninguno de los dos países, por lo que se puede afirmar que tanto hombres como mujeres obtienen puntuaciones similares.

Tabla 2.

Diferencias en el Autoconcepto Académico, Motivación Escolar e Implicación Escolar según el país

\begin{tabular}{|c|c|c|c|c|c|}
\hline País & & $\mathbf{N}$ & M (DT) & $\mathbf{T}$ & $\mathrm{p}$ \\
\hline Autoconcepto Académico Verbal & $\begin{array}{l}\text { República Dominicana } \\
\text { España }\end{array}$ & $\begin{array}{l}567 \\
534\end{array}$ & $\begin{array}{l}16,91(3,38) \\
14,86(3,34)\end{array}$ & 10,12 & ,000 \\
\hline $\begin{array}{l}\text { Autoconcepto Académico } \\
\text { Matemático }\end{array}$ & $\begin{array}{l}\text { República Dominicana } \\
\text { España }\end{array}$ & $\begin{array}{l}567 \\
534\end{array}$ & $\begin{array}{l}14,02(3,83) \\
12,90(4,58)\end{array}$ & 4,40 & , 000 \\
\hline Autoconcepto Académico & $\begin{array}{l}\text { República Dominicana } \\
\text { España }\end{array}$ & $\begin{array}{l}567 \\
534\end{array}$ & $\begin{array}{l}30,93(5,53) \\
27,76(5,22)\end{array}$ & 9,78 & ,000 \\
\hline Metas extrínsecas & $\begin{array}{l}\text { República Dominicana } \\
\text { España }\end{array}$ & $\begin{array}{l}567 \\
534\end{array}$ & $\begin{array}{l}22,01(5,15) \\
16,55(5,25)\end{array}$ & 17,40 & ,000 \\
\hline Metas intrínsecas & $\begin{array}{l}\text { República Dominicana } \\
\text { España }\end{array}$ & $\begin{array}{l}567 \\
534\end{array}$ & $\begin{array}{l}16,40(3,53) \\
15,32(2,72)\end{array}$ & 5,68 & ,000 \\
\hline Motivación Escolar & $\begin{array}{l}\text { República Dominicana } \\
\text { España }\end{array}$ & $\begin{array}{l}567 \\
534\end{array}$ & $\begin{array}{l}164,80(20,81) \\
145,68(20,48)\end{array}$ & 13,34 & ,000 \\
\hline Implicación comportamental & $\begin{array}{l}\text { República Dominicana } \\
\text { España }\end{array}$ & $\begin{array}{l}567 \\
534\end{array}$ & $\begin{array}{l}19,72(1,91) \\
18,90(1,65)\end{array}$ & 7,65 &, 000 \\
\hline Implicación emocional & $\begin{array}{l}\text { República Dominicana } \\
\text { España }\end{array}$ & $\begin{array}{l}567 \\
534\end{array}$ & $\begin{array}{l}24,36(4,25) \\
21,60(3,49)\end{array}$ & 11,80 & ,000 \\
\hline Implicación cognitiva & $\begin{array}{l}\text { República Dominicana } \\
\text { España }\end{array}$ & $\begin{array}{l}567 \\
534\end{array}$ & $\begin{array}{l}28,95(6,22) \\
24,12(4,65)\end{array}$ & 14,66 & ,000 \\
\hline
\end{tabular}

Se procede a realizar una comparativa de las posibles diferencias en todas las variables estudiadas en función del país, encontrándose que en todas ellas es el alumnado de República Dominicana el que puntúa significativamente por encima del alumnado universitario español con una significación $\mathrm{p}<, 001$ (ver tabla 2).

A continuación un segundo objetivo, busca aclarar la direccionalidad de la relación entre autoconcepto académico, motivación escolar e implicación escolar en estudiantes universitarios de República Dominicana y España. A tal fin se llevan a cabo regresiones lineales, primero de las variables de autoconcepto académico y motivación escolar sobre cada dimensión de la implicación escolar (tablas 3 a 5) y posteriormente de la implicación escolar sobre el autoconcepto académico y la implicación escolar sobre la motivación escolar (tablas 6 y 7) respectivamente.

En la tabla 3 se presentan los datos de regresión del autoconcepto académico y la motivación escolar sobre la implicación conductual. Los resultados indican que la varianza de la implicación conductual viene explicada en un $13 \%\left(R^{2}=, 133\right)$ por ambas variables, mientras que en España esa capacidad explicativa es considerablemente menor, situándose en un $4,1 \%\left(R^{2}=, 041\right)$.

Tabla 3.

Capacidad Predictiva del Autoconcepto Académico y la Motivación Escolar sobre Implicación Comportamental según ambos países

\begin{tabular}{|c|c|c|c|c|c|c|c|c|c|c|c|c|}
\hline \multicolumn{9}{|c|}{ República Dominicana } & \multicolumn{4}{|c|}{ España } \\
\hline & \multicolumn{2}{|c|}{ Modelo } & \multicolumn{4}{|c|}{ Coeficiente de Regresión } & \multicolumn{2}{|c|}{ Modelo } & \multicolumn{4}{|c|}{ Coeficiente de Regresión } \\
\hline & $\mathrm{R}$ & $R^{2} \mathrm{C}$ & B.N. & B.E. & $\mathrm{T}$ & $\mathrm{P}$ & $\mathrm{R}$ & $R^{2} \mathrm{C}$ & B.N. & B.E. & $t$ & $\mathrm{P}$ \\
\hline Cons & & & & & 28,25 &, $000^{* * *}$ & & & & & 48,62 &, $000^{* * *}$ \\
\hline A.A.V. & & & ,083 & ,147 & 3,62 &, $000^{* *}$ & & & & & & \\
\hline A.A.M. & & & ,060 & 121 & 3,01 &, $003^{* *}$ & & & & & & \\
\hline $\begin{array}{l}\text { M.E. } \\
\text { M.I. }\end{array}$ & ,371 & 133 & & & & & ,207 & ,041 & & & & \\
\hline M.G. & & & ,068 & ,262 & 6,48 &, $000 * * *$ & & & , 053 & ,207 & 4,89 &, $000^{* * *}$ \\
\hline ANOVA & F 29 & & & (p), & & & F 23, & & & $(\mathrm{p})$, & & \\
\hline
\end{tabular}

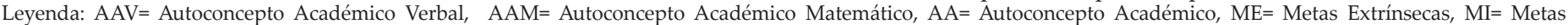
Intrínsecas, MG= Motivación General.

También se puede observar que, mientras que en República Dominicana tienen capacidad predictiva los dos tipos de autoconcepto académico (verbal: $t=3,62, p<, 001$; matemático: $t=$
$3,01, p<, 01)$ junto con la motivación general $(t=6,48, p<, 001)$, en España es la motivación general la única variable con algún tipo de capacidad de influencia $(t=4,89, p<, 001 ; \beta=, 207)$ sobre 
la implicación conductual o comportamental. Ahora bien, es en España donde el peso predictivo de la motivación es algo mayor $(\beta=, 207$ vs $\beta=, 164)$ que en el otro país, si bien en República Dominicana es el autoconcepto matemático, comparativamente con el verbal y la motivación general, el que mayor peso predictivo presenta.

En cuanto a la capacidad predictiva del autoconcepto académico y la motivación escolar sobre la implicación emocional, los datos de la regresión lineal correspondiente, se presentan en la tabla 4. En este caso, de nuevo en España la variable motivación general es la única que puede predecir este tipo de implicación $(t=4,76, p<, 001)$. Ahora bien en República Dominicana lo hacen el autoconcepto académico matemático $(t=5,57, p<, 001)$, la motivación intrínseca $(t=3,00, p<, 01)$ y la motivación general $(t=2,76$, $p<, 01)$, siendo de las tres el autoconcepto matemático la que más capacidad predictiva logra $(\beta=, 220)$ seguida de la motivación intrínseca $(\beta=, 182)$ y la general $(\beta=, 164)$. Todo ello hace que la varianza de la implicación emocional en República Dominicana esté explicada en un $18 \%\left(R^{2}=, 182\right)$ por el autoconcepto académico y la motivación escolar, frente a un $9 \%$ en España $\left(R^{2}=, 092\right)$.

Tabla 4.

Capacidad Predictiva del Autoconcepto Académico y la Motivación Escolar sobre Implicación Emocional según ambos países

\begin{tabular}{|c|c|c|c|c|c|c|c|c|c|c|c|c|}
\hline & \multicolumn{6}{|c|}{ República Dominicana } & & & & \multicolumn{3}{|c|}{ España } \\
\hline & \multicolumn{2}{|c|}{ Modelo } & \multicolumn{4}{|c|}{ Coeficiente de Regresión } & \multicolumn{2}{|c|}{ Modelo } & \multicolumn{4}{|c|}{ Coeficiente de Regresión } \\
\hline & $\mathrm{R}$ & $R^{2} \mathrm{C}$ & B.N & B. E & $\mathrm{T}$ & $p$ & $\mathrm{R}$ & $R^{2} \mathrm{C}$ & B.N & B.E. & $\mathrm{t}$ & $\mathrm{P}$ \\
\hline Cons. & & & & & 13,90 &, $000^{* * *}$ & & & & & 8,61 &, $000^{* * *}$ \\
\hline \multicolumn{13}{|l|}{ A.A.V. } \\
\hline A.A.M. & & & ,245 & ,220 & 5,57 &, $000^{* * *}$ & & & & & & \\
\hline $\begin{array}{l}\text { M.E. } \\
\text { M.I. }\end{array}$ & ,431 & 182 & 219 & 182 & 3,00 &, $003^{* *}$ & ,308 & ,092 & & & & \\
\hline M.G. & & & 095 & 164 & 2,76 &, $006^{* *}$ & & & 134 & ,246 & 4,76 &, $000^{* * *}$ \\
\hline ANOVA & \multicolumn{2}{|c|}{ F 42,903} & & \multicolumn{3}{|c|}{ (p),, $000 \mathrm{~b}$} & \multicolumn{2}{|c|}{ F 27,908} & & \multicolumn{3}{|c|}{ (p),, $000 \mathrm{~b}$} \\
\hline
\end{tabular}

${ }^{* * *} \mathrm{p}<.001,{ }^{* *} \mathrm{p}<.01,{ }^{*} \mathrm{p}<.05$

Leyenda: $\mathrm{AAV}=$ Autoconcepto Académico Verbal, $\mathrm{AAM}=$ Autoconcepto Académico Matemático, AA= Autoconcepto Académico, ME $=$ Metas Extrínsecas, MI= Metas Intrínsecas, MG= Motivación General.

Finalmente el análisis relativo a la capacidad predictiva del autoconcepto académico y la motivación sobre el último tipo de implicación, concretamente la implicación cognitiva, se expone en la tabla 5.

Tabla 5.

Capacidad Predictiva del Autoconcepto Académico y la Motivación Escolar sobre Implicación Cognitiva según ambos países

\begin{tabular}{|c|c|c|c|c|c|c|c|c|c|c|c|c|}
\hline \multicolumn{7}{|c|}{ República Dominicana } & & \multicolumn{5}{|c|}{ España } \\
\hline & \multicolumn{2}{|c|}{ Modelo } & \multicolumn{4}{|c|}{ Coeficiente de Regresión } & \multicolumn{2}{|c|}{ Modelo } & \multicolumn{4}{|c|}{ Coeficiente de Regresión } \\
\hline & $\mathrm{R}$ & $R^{2} \mathrm{C}$ & B.N. & B.E. & $\mathrm{T}$ & $\mathrm{p}$ & $\mathrm{R}$ & $R^{2} \mathrm{C}$ & B.N. & B.E. & $\mathrm{t}$ & $\mathrm{P}$ \\
\hline Cons. & & & & & 13,48 &, $000^{* * *}$ & & & & & 8,53 &, $000^{* * *}$ \\
\hline A.A.V. & & & & & & & & & ,236 & , 170 & 4,11 &, $000^{* * *}$ \\
\hline A.A.M. & & & ,229 & ,206 & 5,17 &, $000^{* * *}$ & & & ,085 &, 084 & 2,02 &, $044^{*}$ \\
\hline $\begin{array}{l}\text { M.E. } \\
\text { M.I. } \\
\text { M.G. }\end{array}$ & ,421 & 173 & ,357 & ,296 & 7,24 &, $000 * * *$ & ,369 & 132 &, 501 & ,293 & 7,12 &, $000 * * *$ \\
\hline ANOVA & F 40, & & & (p), 0 & & & F 27, & & & (p), & & \\
\hline
\end{tabular}

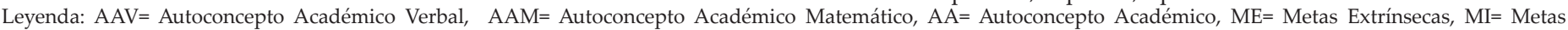
Intrínsecas, $\mathrm{MG}=$ Motivación General.

En este caso, los resultados varian considerablemente si se comparan con los otros dos tipos de implicación anteriores. Así, en España la motivación general pasa a ser una variable no predictiva y aparecen con esta capacidad los dos tipos de autoconcepto académico (verbal: $t=8,53, p<, 001$; matemático: $t=2,02, p$ $<, 05)$ y la motivación intrínseca $(t=7,12 ; p<, 001)$; y en República Dominicana únicamente revelan tal capacidad predictiva el autoconcepto matemático $(t=5,17 ; p<, 001)$ y la motivación intrínseca $(t=7,24 ; p<, 001)$. En ambos países es la motivación intrínseca la que más efecto predictivo presenta $\left(\beta_{\text {República }}=, 296 ; \beta_{\text {España }}=, 293\right)$.
Ahora bien, mientras que en República el autoconcepto matemático logra un buen peso predictivo $(\beta=, 206)$ en España se queda con una $\beta=, 084$, siendo más elevado la del verbal $(\beta=, 236)$.

Una vez examinados los datos resultantes de la influencia estadística del autoconcepto académico y la motivación sobre los tres tipos de implicación, queda revisar la influencia de las variables hacia la otra dirección: de los tipos de implicación escolar sobre el autoconcepto académico (tabla 6) y la motivación escolar (tabla 7). 
Tabla 6.

Capacidad Predictiva de la Implicación Escolar sobre el Autoconcepto Académico según ambos países

\begin{tabular}{|c|c|c|c|c|c|c|c|c|c|c|c|}
\hline & \multicolumn{5}{|c|}{ República Dominicana } & \multicolumn{6}{|c|}{ España } \\
\hline & Modelo & \multicolumn{4}{|c|}{ Coeficiente de Regresión } & \multicolumn{2}{|c|}{ Modelo } & \multicolumn{4}{|c|}{ Coeficiente de Regresión } \\
\hline & $\begin{array}{ll}\mathrm{R} & R^{2} \mathrm{C}\end{array}$ & B.N & B.E & $\mathrm{T}$ & $p$ & $\mathrm{R}$ & $R^{2} \mathrm{C}$ & B.N & B.E & $t$ & $\mathrm{P}$ \\
\hline Cons. & & & & 5,16 &, $000^{* * *}$ & & & & & 5,92 &, $000^{* * *}$ \\
\hline I. Co. & & ,436 & 150 & 3,61 &, $000^{* * *}$ & & & ,318 & 100 & 2,24 &, $025^{*}$ \\
\hline I. E. & 174 & 148 & ,114 & 2,48 &, $013^{* *}$ & 235 & ,050 & & & & \\
\hline I. C. & & 246 & 277 & 6,30 &, $000^{* * *}$ & & & 210 & 187 & 4,29 &, $000^{* * *}$ \\
\hline ANOVA & F40,614 & & & p), 00 & & F 10 & & & (p), 0 & & \\
\hline
\end{tabular}

Leyenda: ICo= Implicación Comportamental, IE= Implicación Escolar, IC= Implicación Cognitiva.

Los resultados de la tabla 6 revelan que en República Dominicana, la varianza del autoconcepto académico explicada por la implicación escolar alcanza casi un $17,5 \%\left(R^{2}=, 174\right)$, obteniendo todas las variables una pendiente poblacional de regresión significativamente distinta de cero, es decir, obteniendo todas ellas capacidad predictiva: implicación comportamental $(t=3,61, p<$ ,001), implicación emocional ( $t=2,48, p<, 05)$ e implicación cognitiva $(t=6,30, p<, 001)$. En España, sin embargo, alcanza solamente un
$5 \%\left(R^{2}=, 050\right)$ y siendo la implicación emocional el único tipo de implicación que no logra alcanzar una pendiente de regresión lo suficientemente elevada como para afirmar que tenga capacidad predictiva. Si se comparan los niveles de peso predictivo Beta Estandarizada en ambos países, se comprueba que siempre es la implicación conductual la que más valor alcanza, seguida de la la cognitiva, y por último la emocional, pero sólo en el caso del país americano.

Tabla 7.

Capacidad Predictiva de la Implicación Escolar sobre la Motivación General según ambos países

\begin{tabular}{|c|c|c|c|c|c|c|c|c|c|c|c|}
\hline \multicolumn{8}{|c|}{ República Dominicana } & \multicolumn{4}{|c|}{ España } \\
\hline & \multicolumn{2}{|c|}{ Modelo } & \multicolumn{4}{|c|}{ Coeficiente de Regresión } & Modelo & \multicolumn{4}{|c|}{ Coeficiente de Regresión } \\
\hline & $\mathrm{R}$ & $R^{2} \mathrm{C}$ & B.N & B.E & $\mathrm{T}$ & $p$ & R $\quad R^{2} \mathrm{C}$ & B.N & B.E & $t$ & $\mathrm{P}$ \\
\hline Cons & & & & & 3,21 &, $001^{* *}$ & & & & 2,881 &, $004^{* *}$ \\
\hline I.Co. & & & 749 & 195 & 4,72 &, $000 * * *$ & & ,424 & 109 & 2,54 &, $011^{*}$ \\
\hline I. E. & ,441 & 190 & 247 & 144 & 3,14 &, $002^{* *}$ & 126 & 428 & ,232 & 5,39 &, $000^{* * *}$ \\
\hline I. C. & & & 281 & 238 & 5,49 &, $000^{* * *}$ & & ,228 & 165 & 3,95 &, $000^{* * *}$ \\
\hline ANOVA & & 45,387 & & (p) , 00 & & & F 26,620 & & (p), & & \\
\hline
\end{tabular}

Leyenda: $\mathrm{ICo}=$ Implicación Comportamental, IE= Implicación Escolar, IC= Implicación Cognitiva.

Por último, los resultados de la tabla 7 revelan que en ambos países todas las variables independientes (los tres tipos de implicación) resultan con capacidad predictiva sobre la motivación general, dando como resultado que en República Dominicana la varianza total explicada de la motivación por parte de la implicación sea del 19\% $\left(R^{2}=, 190\right)$ frente al $12,5 \%$ en España $\left(R^{2}=, 126\right)$. Ahora bien, el nivel de capacidad predictiva de cada uno de los tipos de implicación varía considerablemente entre países. Así, en el país americano es la implicación cognitiva la que más nivel de motivación puede predecir, $(\beta=, 238)$ seguida de la cognitiva $(\beta=, 195)$ y la emocional $(\beta=, 144)$, mientras que en España la que mayor peso predictivo alcanza es la implicación emocional $(\beta=, 232)$, seguida de la conductual $(\beta=, 109)$ y finalmente la cognitiva $(\beta=, 165)$.

En síntesis, se puede concluir que la implicación escolar predice el autoconcepto académico y la motivación escolar en ambos países, siendo República Dominicana donde mayor capacidad predictiva muestra. De igual forma se puede observar que la implicación comportamental y emocional se pueden predecir en mayor grado en República Dominicana por medio del autoconcepto académico. Sin embargo, la dimensión cognitiva lo hace en España. En cuanto a la motivación escolar sobre la implicación se observa que posee una capacidad predictiva en algunas dimensiones en ambos países.

\section{Discusión}

A partir del primer objetivo planteado para esta investigación el cual busca identificar las diferencias en el autoconcepto académico, motivación escolar e implicación escolar en función del sexo y el país, se puede comprobar que existen diferencias en ambos países del autoconcepto académico matemático a favor de los hombres y del verbal a favor de las chicas, esto resulta contradictorio con estudios realizados en ambos países que afirman que las mujeres poseen mejor concepto de sí (Barca et al., 2011) o que los hombres poseen mayor autoconcepto (Fernández-Zabala et al., 2015). Es decir, las mujeres se muestran mejor en el área social y los hombres con la racionalidad, tal y como presenta los estereotipos sociales de acuerdo al género (Inglés et al., 2009).

Según Cerezo y Casanova (2004) las mujeres poseen una mayor motivación intrínseca, contradiciendo los resultados de esta investigación que no encontró diferencia alguna. A su vez, las chicas se encuentran más motivadas en general (Núñez et al., 1998), confirmando lo encontrado en España donde si existe diferencia a favor de las mujeres.

En cuanto a la implicación escolar se pudo comprobar que existen diferencias significativas en algunas de sus dimensiones, tal es el caso de la implicación comportamental en República Dominicana y en España la comportamental y emocional. Siendo las mujeres las que mayores puntuaciones alcanzan, esto concuerda 
con lo apuntado por Cava, Buelga, Musitu y Murgui (2010) y Hernando, Oliva y Pertegal (2013) quienes sugieren que los chicos suelen estar implicados en menor intensidad en la vida escolar.

De igual forma, se intenta aclarar la direccionalidad de la relación entre el autoconcepto académico, la motivación escolar y la implicación escolar en ambos países, siendo este el segundo y último objetivo que plantea esta investigación. Los resultados demuestran que la implicación escolar predice el autoconcepto académico y la motivación escolar en ambos países. A su vez, hay quienes señalan que el autoconcepto académico (verbal y matemático) muestra relación directa con la implicación escolar (Bush, 2005; Ramos-Díaz, Rodríguez-Fernández, Ros y Antonio-Agirre, 2017), esto coincide con los datos arrojados en este estudio donde los tres componentes de la implicación muestran predicción con las dimensiones del autoconcepto académico en la República, sin embargo, en España solo en la cognitiva.

En cuanto a la influencia de la motivación escolar, se puede observar que en ambos países existe capacidad predictiva sobre la implicación escolar aunque no en todas sus dimensiones. Estos datos son confirmados en distintos estudios que señalan que a mejor implicación, mejor autoconcepto y motivación (Bempechat, 2004; Hong, 2001). Así, como otros estudios que aseguran que mientras mejor sea el autoconcepto y la motivación, el nivel de implicación en la escuela será mejor (Clarks et al., 2000; Dettmers et al., 2010; Pan et al., 2013).

Según Ramo (2003), la motivación intrínseca es la más estudiada debido que es la que mayor influencia ejerce sobre la implicación escolar, sin embargo, en este estudio se pudo comprobar que solo ejerce influencia sobre la implicación emocional y cognitiva. Por su parte, la metas extrínsecas no es significativa en ninguna dimensión de la implicación, contradiciendo así lo encontrado en otra investigación donde afirman que el alumnado que realiza deberes escolares se implica no sólo por el deseo que les provoca o el interés de hacerlo, sino por deseos de agradar e incluso el temor de ser castigados (Walker, Hoover-Dempsey, Whetselm y Grenn, 2004).

A manera general, cabe señalar que de estas tres variables solo ha sido estudiada la motivación en la República Dominicana (Fernández, Arnaiz y Barca, 2009), por tal razón se cree que este estudio aporta nuevas informaciones para poder apalear cualquier duda en cuanto a que factores trabajar para una mejor implicación. Es bueno destacar que en esta investigación se evidencia la existencia de diferencias en cada dimensión de los tres constructos en función del país a favor de la República Dominicana. Esto podría ser debido a que los motivos que llevan a estudiar al alumnado de este país son distintos a uno de España. De igual forma se puede entender que existe una relación importante de una variable sobre otras.

Cabe señalar que como limitación de este estudio se encuentra la naturaleza transversal, debido a esto es imposible obtener una visión más completa de la evolución que pueda existir entre estos estudiantes y su implicación en la escuela. Sin embargo, este estudio ha abierto una línea de investigación que en un futuro podría ser utilizada para la realización de un estudio longitudinal y así aclarar si con el tiempo en esta implicación se produce algún cambio.

Asimismo, otra clara limitación es el amplio rango de edad del estudiantado universitario de República Dominicana, lo que dificulta en cierta medida la comparación de los resultados. Ahora bien, ese amplio rango de edad es reflejo de la realidad social de las universidades existentes en ese país, donde por razones económicas habitualmente el alumnado abandona en secundaria sus estudios para retomarlos muchos años después, cuando su situación económica es más estable y les permite hacer frente a los gastos que supone el estudio de una carrera universitaria. No obstante, de cara a futuras investigaciones resultaría interesante incluir esta variable como una covariable que permitiera examinar: a) si las diferencias de edad entre los universitarios dominicanos conllevan diferencias en la implicación académica, en la motivación escolar y en el autoconcepto académico, así como en las influencias relacionales existentes entre ellas; b) si los patrones explicativos extraídos en este estudio se mantienen a pesar de la inclusión de esta covariable.

De igual forma, los datos obtenidos del presente estudio poseen implicaciones educativas a tener en cuenta en las intervenciones psicoeducativas orientadas a la mejora de la implicación escolar y las variables psicológicas. Conocer la capacidad predictiva de la implicación sobre estas variables permitirá desarrollar estrategias que potencialicen estos factores propios de la implicación y mejorar en el autoconcepto académico y la motivación escolar, pero también es de considerar el papel de la escuela para fomentar una educación centrada en la auto aceptación de cada estudiante y lograr una buena adaptación escolar. Crear un ambiente adecuado, fomentar el trabajo en grupo y orientar al alumnado a involucrarse más en la escuela, permitirá que estos logren alcanzar el funcionamiento óptimo en el entorno educativo (Motti-Stefadini y Masten, 2013).

\section{Referencias}

Amezcua, J., y Pichardo, M. (2000). Diferencias de sexo en autoconcepto en sujetos adolescentes. Anales de Psicología, 16(2), 207-214.

Barca, A., Fernández, A., y Mejía, R. (2011). Autoconcepto y enfoques de aprendizaje: sus efectos en el rendimiento académico en alumnado universitario de República Dominicana. Revista Galego-Portuguesa de Psicoloxía e Educación, 19(2), 1138-1663.

Bempechat, J. (2004). The motivational benefits of homework: A social-cognitive perspective. Theory Into Practice, 43(3), 189196.

Birch, S. H., y Ladd, G. W. (1996). Interpersonal relationships in the school environment and children's early school adjustment: The role of teachers and peers.

Blumenfeld, P., Fredricks, J., y Paris, A. (2004). School engagement: potencial of the concept, state of the evidence. Review of Educational Research, 74, 59-109.

Bush, E. S. (2005). Peer rejection, negative peer treatment, and school adjustment: Self-concept and classroom engagement as mediating processes. Journal of School Psychology, 43(5), 407-424. doi:10.1016/j.jsp.2005.09.001

Cava, M. J., Buelga, S., Musitu, G., \& Murgui, S. (2010). Violencia escolar entre adolescentes y sus implicaciones en el ajuste psicosocial: un estudio longitudinal. Revista de psicodidáctica, 15(1).

Cerezo, M.T., y Casanova, P. F. (2004). Diferencias de género en la motivación académica de los alumnos de Educación Secundaria Obligatoria. Jaén. Revista Electrónica de Investigación Psicoeducativa, 2, 97-112.

Christenson, S. L., Reschly, A. L. y Wylie, C. (2012). Handbook of research on student engagement. New York, NY, US: Springer Science. doi:10.1007/978-1-4614-2018-7

Clark, A., Clemes, H. y Bean, R. (2000). Cómo desarrollar la autoestima en adolescentes. Madrid: Editorial Debate.

Corno, L. (2000). Looking at homework differently. Elementary School Journal, 100(5), 529-548.

Deci, E. L., y Ryan, R. M. (2000). The "what" and "why" of goal pursuits: Human needs and the self-determination of behaviour. Psychological Inquiry, 11, 227- 268. 
Dettmers, S., Trautwein, U., Lüdtke, O., Kunter, M., y Baumert, J. (2010). Homework works if homework quality is high: using multilevel modeling to predict the development of achievement in mathematics. Journal of Educational Psychology, 102, 467-482. http://dx.doi.org/10.1037/a0018453

Dimont, C. G. (1990). Self-concept and school adjustment among siblings of mentally retarded, learning disabled and nonhandicapped children (doctoral dissertation). George Washington University, Estados Unidos.

Fernández, A., Arnaiz, P., y Barca, A. (2009). Motivación, aprendizaje y rendimiento académico en estudiantes de primer año de universidad en la República Dominicana (tesis doctoral). Universidad de Murcia, España.

Fernández-Zabala, A., Goñi, E., Rodríguez-Fernández, A., y Goñi, A. (2015). Un nuevo cuestionario en castellano con escalas de las dimensiones del autoconcepto. Revista Mexicana de Psicología, 32(2).

Fuentes, M. C., García, F., Gracia, E. y Lila, M. (2011). Autoconcepto y ajuste psicosocial en la adolescencia. Psicothema, 23(1), 7-12.

Garaigordobil, M., y Durá, A. (2006). Las relaciones del autoconcepto y la autoestima con la sociabilidad, la seguridad emocional y la responsabilidad en adolescentes de 14 a 17 años. Análisis y Modificación de Conducta, 32, 37-64.

García, F. \& Musitu, G. (2009). Manual del AFA 5. Autoconcepto forma 5. Madrid: TEA.

González, M. C., Roces, C., y Tourón, J. E. (1995). Validación preliminar del CEAM II (Cuestionario de Estrategias de Aprendizaje y Motivación II). Psicológica, 16(3), 347-366.

González, A. y Verónica-Paoloni, P. (2015). Implicación y rendimiento en Física: el papel de las estrategias docentes en el aula, y el interés personal y situacional. Revista de Psicodidáctica, 20, 25-45. doi:http:10.1387/RevPsicodidact.11370

Hay, I., Ashman, A., y Van-Kraayenoord, C. (1998). The educational characteristics of students with high or low self-concept. Psychology in the Schools, 35, 391-400.

Hernando, A., Oliva, A., y Pertegal, M. (2013). Diferencias de género en los estilos de vida de los adolescentes. Psychosocial intervention, 22(1), 15-23.

Hong, E. (2001). Homework style, homework environment, and academic achievement. Learning Environments Research, 4(1), 7-23.

Inglés, C., Martínez-Monteagudo, M., García-Fernández, J., Valle, A. y Castejón, J. (2014). Perfiles de orientaciones de metas y autoconcepto de estudiantes de Educación Secundaria. Revista de Psicodidáctica, 20, 99-116. doi:10.1387/RevPsicodidact.1023

Inglés, C. J., Pastor, Y., Torregrosa, M. S., Redondo, J., y García, J. M. (2009). Diferencias en función del género y del curso académico en dimensiones del autoconcepto: Estudio con una muestra de adolescentes españoles. Anuario de Psicología, 40(2), 271-288.

Martínez-Antón, M., Buelga, S., y Cava, M. J. (2007). La satisfacción con la vida en la adolescencia y su relación con la autoestima y el ajuste escolar. Anuario de psicología/The UB Journal of psychology, 38(2), 293-303.
Motti-Stefanidi, F., y Masten, A.S. (2013). School success and school engagement of immigrant children and adolescents: A risk and resilience developmental perspective. European Psychologist, 18(2), 126-135. doi:10.1027/1016-9040/a000139

Núñez, J.C., González-Pienda, J.A., García, M.S., González-Pumariega, S. y García, S.I. (1998). Estrategias de aprendizaje en estudiantes de 10 a 14 años y su relación con los procesos de atribución causal, el autoconcepto y las metas de estudio. Estudios de Psicología, 59, 65-85.

Pan, I., Regueiro, B., Ponte, B., Rodríguez, S., Piñeiro. I., y Valle, A. (2013). Motivación, implicación en los deberes escolares y rendimiento académico. Aula Abierta, 41(3), 13-22.

Pintrich, P. R., Roeser, R. W., y De Groot, E. A. (1994). Classroom and individual differences in early adolescents motivation and self-regulated learning. Journal of Early Adolescence, 14, 139-161.

Ramo, A. (2003). La Motivación en el estudio. Especial desde España para El Cronista Regional. 19/12/2003.

Ramos-Díaz, E., Rodríguez-Fernández, A., y Revuelta, L. (2016). Validation of the Spanish Version of the School Engagement Measure (SEM). The Spanish Journal of Psychology, 19, 1-9. doi: $10.1017 /$ sjp.2016.94

Ramos-Díaz, E., Rodríguez-Fernández, A., Fernández-Zabala, A., Revuelta, L., y Zuazagoitia, A. (2016). Apoyo social percibido, autoconcepto e implicación escolar de estudiantes adolescentes. Revista de Psicodidáctica, 21(2), 339-356.

Ramos-Díaz, E., Rodríguez-Fernández, A., Ros, I., \& Antonio-Agirre, I. (2017). Implicación escolar y autoconcepto multidimensional en una muestra de estudiantes de secundaria. Revista Complutense de Educación, 28(4), 113.

Ros, I. (2009). La implicación del estudiante con la escuela. Revista de Psicodidactica, 14(1), 79-92.

Rodríguez-Fernández, A., Droguett, L. y Revuelta, L. (2012). Ajuste escolar y personal en la adolescencia: El papel del autoconcepto académico y del apoyo social percibido. Revista de Psicodidáctica, 17, 397-414. doi: 0.1387/Rev.Psicodidact.3002

Ruiz de Azúa, U. (2012). DBHko ikasleen euskararekiko jarrera linguistikoak, motibazioa eta errendimendu akademikoa. [Actitudes lingüísticas hacia el euskera, motivación y rendimiento académico de estudiantes de Educación Secundaria Obligatoria]. Tesis Doctoral. Lejona: UPV/EHU.

Shavelson, R. J., Hubner, J.J., Stanton, J.C. (1976). Self concept:validation of construct interpretations.Review of educational Research,46,407-441

Simon-Morton, B. y Chen, R. (2009). Peer and parent Influences on school engagement among early adolescents. Youth \& Society, 41(1), pp. 3-25.

Vallerand, R. J., y Bissonnette, R. (1992). Intrinsic, extrinsic, and amotivational styles as predictors of behavior: A prospective study. Journal of Personality, 60, 599-620.

Walker, J. M., Hoover-Dempsey, K. V., Whetsel, D. R., y Green, C. L. (2004). Parental involvement in homework. A Review of Current Research and Its Implications for teachers, After School Program Staff, and Parent Leaders. Harvard Family Research Project.

Xu, J., y Corno, L. (1998). Case studies of families doing third-grade homework. The Teachers College Record, 100(2), 402-436. 